\title{
Systems biology intertwines with single cell and $\mathrm{Al}$
}

\author{
Yong Wang ${ }^{1,2,4^{*}}$, Xiang-Sun Zhang ${ }^{1}$ and Luonan Chen ${ }^{3,4^{*}}$ \\ From The 12th International Conference on Computational Systems Biology (ISB 2018) \\ Guiyang, China. 18-21 August 2018
}

\begin{abstract}
A report of the 12th International Conference on Systems Biology (ISB2018), 18-21 August, Guiyang, China.
\end{abstract}

Keywords: Systems biology, Network, Data integration

\section{Background}

To study the complex genotype phenotype mapping, there are two distinct scientific paradigms. The reductionist approach decomposes the phenotypic variability step by step with the assumption that the complex life system is constructed from the additive effect of their molecules. In contrast, systems biology choses to represent the whole system by investigating the networks, which are the large scale interactions of many molecules in a systematic and simultaneously way.

The recent trend is that the two approaches are becoming increasingly complementary to each other and intertwined. The recent years witnessed the breakthrough of single cell sequencing technology, which enable to look into complex interactions at systems level inside reductive single cell level. Single cell analysis has been beyond the capabilities of 'Omics' technologies. This is rapidly changing with the recent examples of single cell genomics, transcriptomics, proteomics, and metabolomics.

Single cell data helps to address the cellular heterogeneity arising from stochastic expression of genes, proteins, and metabolites is a fundamental principle of cell biology. Single cell analysis is the new frontier in Omics, and single cell Omics has the potential to transform

\footnotetext{
* Correspondence: ywang@amss.ac.cn; Inchen@sibs.ac.cn

${ }^{1}$ CEMS, NCMIS, MDIS, Academy of Mathematics and Systems Science, Chinese Academy of Sciences, Beijing 100190, China

${ }^{3}$ Key Laboratory of Systems Biology, SIBS-Novo Nordisk Translational Research Centre for PreDiabetes, Shanghai Institutes for Biological Sciences, Chinese Academy of Sciences, Shanghai 200031, China

Full list of author information is available at the end of the article
}

systems biology through new discoveries derived from cellular heterogeneity [1].

In addition to the single cell level, another trend is that systems biology is boosted by the artificial intelligence (AI) development. The reason is simple. In the era of big biological data, systems biology needs to mine biological insights from genome and transcriptome expression generated using next-generation sequencing and other technologies and further integrate with proteomes and metabolomes data. We noticed that big data analysis is required in various other fields such as machine learning and AI. For example, deep-learning algorithms take raw features from an extremely large, annotated data set, such as a collection of images or genomes, and use them to create a predictive tool based on patterns buried inside. These algorithms have recently shown impressive results across a variety of domains. The ideas and methods developed there can be borrowed for systems biology studies. Biology and medicine are data-rich disciplines, but the data are complex and often ill-understood. Hence, deep learning techniques may be particularly well suited to solve problems of these fields [2]. We can expect deep learning methods to provide valuable means for speeding up or aiding human investigation in future systems biology study.

Along with those new trends, this energetic interdisciplinary field has kept attracting excellent scientists and making significant progresses to convert the biological data to fundamental insights in biology and medicine. Our International Conference on Computational Systems Biology (ISB), launched 11 years ago [3-8], 
continues to serve as a high-quality platform and brought many researchers and students to freely exchange ideas. The 12th International Conference on Computational Systems Biology (ISB2018) was successfully co-organized by Computational Systems Biology Society of ORSC, Molecular Systems Biology Division of CSBMB, SIBS Guian Bio-Med Big Data Center, Guizhou Normal University School of Life Sciences, Guizhou Science and Technology Information Center. We hope that the joint efforts of societies, funding agencies, research institutes, and universities will further push the development of computational methodologies, algorithms, and software in systems biology.

\section{Meeting report}

The 12th International Conference on Computational Systems Biology (ISB 2018) was held in Guiyang, China, August 18-21, 2018. The conference is supported and sponsored by Academy of Mathematics and Systems Sciences of CAS (AMSS), Shanghai Institutes for Biological Sciences of CAS (SIBS), National Natural Science Foundation of China (NSFC), Functional Genome Informatics and Systems Biology Society of CSCB, Systems Biology Technical Committee of IEEE SMC Society.

ISB2018 attracted great leading scientists working in biology, physics, mathematics and computer science, optimization, statistics, and many other mathematical methods. Following the successful ISB conferences series since 2007, ISB 2018 aims to extend the international forum for scientists, researchers, educators, and practitioners to exchange ideas and approaches, to present research findings and state-of-the-art solutions in this interdisciplinary field, including theoretical methodology development and its applications in biosciences and researches on various aspects of computational systems biology.

One hundred and five submissions to ISB2018 cover wide range of computational systems biology. Moreover, the reviewers from the Program Committee of ISB2018 selected 13 papers to be recommended for a special issue in BMC Systems Biology after significant extension of their original submission. Each submission has been peer reviewed and evaluated by three independent reviewers on the quality, originality, soundness, and significance of its contributions. Here we focus on some of the highlights of the meeting by categorizing and briefly introducing these selected papers.

Weber et al. aims to statistically infer the effective regulatory factors that are responsible for the gene expression differentiation with the help from the binding data between factors and genes. They propose a definition of binding strength based on a probability model to allow the inferences to be carried out in silico. Wang et al. propose a new generalized gene co-expression analysis algorithm via subspace clustering that can identify biologically meaningful gene co-expression modules with genes that are not all highly correlated. Hao et al. carried out an integrated analysis of lipid metabolism in cancers originating from different tissues using multiple omics data from TCGA and may provide novel insight into the understanding of tumorigenesis and progression. $\mathrm{Hu}$ et al. proposed a novel motif discovery algorithm based on support vector machine (MD-SVM) to learn a discriminative model for TF binding sites under a computational framework of multiple instance learning (MIL). Shih et al. proposed a voting mechanism-based (LE) prediction system to analyze any two differently clustered pathogen groups, allowing both conserved and exclusive LEs to be identified simultaneously. Liang et al. developed a single cell-based multi-scale spatio-temporal model to simulate vascular tumor growth and drug response, based on VEGFR signaling pathways, EGFR signaling pathway and cell cycle as well as several micro environmental factors that determine cell fate switches in a temporal and spatial context. Xu et al. provided a therapeutic effect recognition strategy is provided based on dynamic network biomarkers (DNB) for CML patients' gene expression data by transforming the undirected interaction network to directed network by employing Granger causality test, followed by the predictors screened with the use of the stepwise character selection algorithm. Aouiche et al. proposed a powerful and versatile framework to identify stage-specific cancer genes and their related dynamic modules by integrating multiple datasets. Zhang et al. employed ten potential computed tomography angiography features to develop a predictive model for intracerebral haemorrhage by employing an experimental design, statistical test, and classification algorithms, which is efficient in predicting the probability of intracerebral haemorrhage. Yu et al. developed a community-driven open-source Ontology of Drug Adverse Events (ODAE) to provide a general representation of ADEs given different conditions and can be used for querying scientific questions. Luo et al. developed a strategy to screen the synergistic combinations of two drug targets in disease networks based on the classification of single drug targets. The method tried to identify the sensitivity of single intervention and then the combination of multiple interventions that can restore the disease network to a desired normal state. Chen et al. proposed a novel model-based algorithm, named VPAC, for accurate clustering of single-cell transcriptomic data through variational projection, which assumes that single-cell samples follow a Gaussian mixture distribution in a latent space.

\section{Abbreviations}

Al: Artificial intelligence; ISB: International Conference on Computational Systems Biology 


\section{Acknowledgements}

We would like to thank all the authors who submitted their manuscripts to this Special Issue. We would also like to thank the reviewers for their invaluable contributions to the peer review process.

\section{Funding}

Publication costs are funded by the Strategic Priority Research Program of the Chinese Academy of Sciences (No.XDB13000000). The authors are also supported by The National Natural Science Foundation of China (NSFC) under Grants nos. 11871463, 61671444, 61621003, 91529303, 31771476,

\section{Availability of data and materials}

The conference information can be found at: http://www.aporc.org/ISB/ 2018/

\section{About this supplement}

This article has been published as part of BMC Bioinformatics Volume 20 Supplement 7, 2019: Selected papers from the 12th International Conference on Computational Systems Biology (ISB 2018). The full contents of the supplement are available online at https://bmcbioinformatics.biomedcentral. com/articles/supplements/volume-20-supplement-7.

\section{Authors' contributions}

YW drafted the manuscript. XSZ and LC read and approved the manuscript. All authors read and approved the final manuscript.

\section{Ethics approval and consent to participate}

Not applicable.

\section{Consent for publication}

Not applicable.

\section{Competing interests}

The authors declare that they have no competing interests.

\section{Publisher's Note}

Springer Nature remains neutral with regard to jurisdictional claims in published maps and institutional affiliations.

\section{Author details}

'CEMS, NCMIS, MDIS, Academy of Mathematics and Systems Science, Chinese Academy of Sciences, Beijing 100190, China. ${ }^{2}$ School of Mathematical Sciences, University of Chinese Academy of Sciences, Beijing 100049, China. ${ }^{3}$ Key Laboratory of Systems Biology, SIBS-Novo Nordisk Translational Research Centre for PreDiabetes, Shanghai Institutes for Biological Sciences, Chinese Academy of Sciences, Shanghai 200031, China. ${ }^{4}$ Center for Excellence in Animal Evolution and Genetics, Chinese Academy of Sciences, Kunming 650223, China.

Published: 1 May 2019

\section{References}

1. Wang D, Bodovitz S. Single cell analysis: the new frontier in 'omics. Trends Biotechnol. 2010;28(6):281-90.

2. Ching T, Himmelstein DS, Beaulieu-Jones BK, et al. Opportunities and obstacles for deep learning in biology and medicine. J R Soc Interface. 2018; 15(141):20170387.

3. Wang Y, Zhang X-S, Chen L. Optimization meets systems biology. BMC Syst Biol. 2010;4(Suppl 2):S1.

4. Wang Y, Zhang X-S, Chen L. Computational systems biology: integration of sequence, structure, network, and dynamics. BMC Syst Biol. 2011;5(Suppl 1):S1.

5. Wang Y, Zhang X-S, Chen L. Modelling biological systems from molecules to dynamical networks. BMC Syst Biol. 2012;6(Suppl 1):S1.

6. Wang $Y$, Zhang X-S, Chen L. Computational systems biology in the big data era. BMC Syst Biol. 2013;7(Suppl 1):S1.

7. Wang $Y$, Zhang $X-S$, Chen L. Understanding biological systems through the lens of data. BMC Syst Biol. 2017;11(Suppl 4):S1.

8. Wang Y, Zhang X-S, Chen L. Integrating data-and model-driven strategies in systems biology. BMC Syst Biol. 2018;12(Suppl 4):S1.

\section{Ready to submit your research? Choose BMC and benefit from:}

- fast, convenient online submission

- thorough peer review by experienced researchers in your field

- rapid publication on acceptance

- support for research data, including large and complex data types

- gold Open Access which fosters wider collaboration and increased citations

- maximum visibility for your research: over $100 \mathrm{M}$ website views per year

At BMC, research is always in progress.

Learn more biomedcentral.com/submissions 